\title{
Diversidad alfa y beta de los artrópodos en diferentes ambientes del Parque Nacional Los Cardones, Salta (Argentina)
}

\author{
María Belén Cava ${ }^{1}$, José Antonio Corronca ${ }^{1} \&$ Alejandro José Echeverría ${ }^{2}$ \\ 1. CONICET-IEBI-FCN (U.N.Sa).Av. Bolivia 5150, Salta, Argentina; belencava@iebi.com.ar, jcorronca@iebi.com.ar \\ 2. IEBI (Instituto para el Estudio de la Biodiversidad de Invertebrados-FCN-U.N.Sa). Av. Bolivia 5150, Salta, Argentina; \\ alejandro.j127@gmail.com
}

Recibido 26-VII-2012. Corregido 25-IV-2013. Aceptado 28-V-2013.

\begin{abstract}
Alpha and beta arthropods diversity from the different environments of Parque Nacional Los Cardones, Salta, Argentina. The essential role of the National Parks is to protect nature, in order to prevent the deterioration and loss of the ecosystem under protection. Very few records about the diversity of arthropods are known from Los Cardones National Park, where three eco-regions are protected: Puna and Monte eco-regions and the High Andean Grassland of the Yungas. Here, we aimed to compare the alpha and beta diversity of arthropods in these eco-regions, and to prove if sites from the same ecoregion, show greater similarity between them in their assemblages, than with sites of the other eco-regions. We also identified arthropod orders with higher species richness, and indicated the families that contribute the most to the registered beta diversity. Three sampling sites were established on each eco-region and the arthropods were sampled using pitfall traps and suction samples. We evaluated the obtained inventory through nonparametric estimators of species richness, and compared diversity among eco-regions through "diversity profiles" and "effective number of species". Beta diversity was assessed by different methods such as the Morisita Index, nonmetric multidimentional scaling analysis, a multiple permutation procedure, and a Similarity Percentage analysis. We recorded $469 \mathrm{spp} / \mathrm{morphospecies}$ and recognized three arthropod orders (spiders, dipterans and hymenopterans) that are diverse and abundant in the Park. Besides, the diversity in Los Cardones National Park was found to be high, but it was observed higher in the High Andean Grassland of the Yungas, and lower in the Puna. The inventory obtained was good, reached up to the $81 \%$ of the species richness estimated by nonparametric estimators. Each eco-region of the park showed a very particular arthropod community that was tested by a multi-response permutation procedure. The species turnover between eco-regions was high, so that the different environments of the protected area are contributing to the maintenance of the regional diversity of arthropods in the park. The assemblages of arthropods belonging to the same eco-region sites showed greater similarity among themselves than with those of more distant sites. This represents the first attempt for biodiversity studies in these areas, but more evaluations are required to detail on the possible climate change and human impacts in the ecosystem. Rev. Biol. Trop. 61 (4): 1785-1798. Epub 2013 December 01.
\end{abstract}

Key words: arthropods, alpha diversity, beta diversity, eco-regions, natural protected area, Argentina.

Uno de los principales problemas ambientales actuales es la aceleración en la tasa de extinción de especies asociada a las actividades humanas, hecho que provoca una pérdida irreversible de la diversidad biológica y que puede tener consecuencias impredecibles para el equilibrio del ambiente, ya que se advierte que muchos servicios de los ecosistemas están declinando de manera acelerada (Purvis \& Hector, 2000). Por ello, la conservación de la diversidad biológica ha llegado a convertirse en una preocupación global y un objetivo ineludible, dedicándose un creciente esfuerzo para entender cómo estas reducciones afectarán el funcionamiento de los ecosistemas (Chapin et al., 1997; Tilman, 1999; Loreau et al., 2001).

La IUCN (1994) define los Parques Nacionales como áreas naturales de tierra y/o mar, designadas para: (a) proteger la integridad ecológica de uno o más ecosistemas para las 
generaciones presentes y futuras, (b) excluir los tipos de explotación u ocupación que sean hostiles al propósito con el cual fue designada el área, y (c) proporcionar una base para las oportunidades espirituales, científicas, educativas, recreativas y turísticas, actividades que deben ser ambiental y culturalmente compatibles. De esta manera, el rol fundamental de los Parques Nacionales es la protección de la naturaleza a partir de políticas que generen y mantengan áreas restringidas y zonas de reserva, que eviten el deterioro y la pérdida del ecosistema. Así, las áreas protegidas representan un instrumento de gestión para equilibrar la conservación y el uso de los recursos naturales (Roux et al., 2008).

La diversidad ha sido separada en diferentes componentes de acuerdo a los fenómenos que son de interés para los ecólogos (Whittaker, 1972). Así, la diversidad alfa mide la riqueza de especies de un ensamble local; mientras que la beta, el grado de diferencia entre las comunidades (Whittaker, Willis \& Field, 2001). La diversidad alfa, de la cual la riqueza de especies es la medida más visible, es quizás el aspecto más estudiado de la diversidad (Cardoso, Borges, Dinis \& Gaspar, 2010). Sin embargo, la diversidad beta, o recambio en la composición de especies entre sitios, es un criterio importante para alcanzar una adecuada representación de la biodiversidad regional en sistemas de áreas protegidas (Kattan et al., 2006). Este concepto es clave para comprender el funcionamiento de los ecosistemas, y para el manejo y conservación de la biodiversidad (Legendre, Borcard \& Peres-Neto, 2005).

Los artrópodos ofrecen un gran potencial como indicadores de la biodiversidad (Gray \& Jongepier, 2008), y son importantes taxa modelo para comparaciones de comunidades dependientes del hábitat (Lassau, Hochuli, Cassis \& Reid, 2005), ya que juegan roles trascendentales en ciertos procesos ecosistémicos, como son la descomposición, la polinización, la depredación y el parasitismo, siendo estas últimas actividades, las que permiten la regulación de los tamaños poblacionales de otras especies (Rivera-García \& Viggers-Carrasco, 1991).
También constituyen un importante recurso alimentario para distintos organismos consumidores, principalmente vertebrados (Gonzales del Solar, Puig, Videla \& Roig, 1997).

Las ecorregiones son grandes áreas relativamente homogéneas, en las que hay diferentes comunidades naturales que tienen en común un gran número de especies y condiciones ambientales (Brown, Martínez-Ortiz, Acerbi \& Concuera, 2006). Tres de ellas están representadas en el Parque Nacional Los Cardones, donde poco se conoce sobre la diversidad de los artrópodos ya que hasta la fecha no se hicieron recolectas ni estudios sistematizados. Los escasos datos de artrópodos en el parque son fragmentados, registrándose dos especies de coleópteros, ocho de himenópteros y 56 de lepidópteros (Moschione, 2009; SIB-APN, 2012), sumado a unas 29 especies de heterópteros recientemente registradas por Cava, Corronca \& Coscarón (2012). Por lo anterior, este trabajo tiene como objetivo contribuir al conocimiento de la biodiversidad de artrópodos de esta área natural protegida, para generar una información de base que pueda ser utilizada para futuros estudios en otras áreas del parque y en ecorregiones del Noroeste Argentino. Así, se quiere comparar la diversidad de artrópodos en las diferentes ecorregiones del parque y probar si los sitios de una misma ecorregión muestran una mayor similitud en sus ensambles de artrópodos que con sitios de otras ecorregiones; existiendo un alto recambio de especies entre ellas. Asimismo, se identifican los órdenes de artrópodos con mayor riqueza de especies, indicando las familias que más aportan a la diversidad beta registrada en el Parque Nacional.

\section{MATERIALES Y MÉTODOS}

Área de estudio: El Parque Nacional Los Cardones se ubica en los departamentos San Carlos y Cachi, Centro-Oeste de la provincia de Salta (Argentina), posee una superficie de 65000 ha entre los 2700 m.s.n.m. (en el fondo del Valle de Tin-Tin) hasta los 5000m.s.n.m. en el cerro Malcante (en el límite Noroeste). En 
él, el clima es árido, seco y cálido, con temperaturas medias de $11^{\circ} \mathrm{C}$ en invierno y $18^{\circ} \mathrm{C}$ en verano, y con precipitaciones que no superan los 200mm anuales (Chebez, 2005). En el parque se encuentran ambientes correspondientes a tres ecorregiones bien diferenciadas: Pastizal Altoandino Nublado de las Yungas (PAN), el Monte Occidental (MO) y la Prepuna-Puna (PP) (Chebez, 2005). El PAN se caracteriza por praderas cerradas dominadas por Alchemilla pinnata Ruiz \& Pavon, Stipa chrysophylla E. Desv., Dichondra microcalyx (Hallier f.) Fabris y Oxalis catamarcensis R. Knuth; mientras que los ambientes del MO son arbustales abiertos y altos donde la vegetación está representada por Flourensia suffrutescens (R.E. Fr.) Blake, Bulnesia schickendantzii Hieron. ex Griseb. y Baccharis polifolia Griseb (Sánchez, 2009). En cambio, la presencia de Echinopsis atacamensis (Phil.) Friedrich \& G.D. Rowley y un arbustal disperso bajo dominado por Junellia longidentata Mold., Baccharis boliviensis (Wedd.) Cabrera, Adesmia horrida Gillies ex Hook. \& Arn., y Salvia gilliesii Benth., es característico de los ambientes de la Prepuna-Puna del parque. A nivel mundial el Parque Nacional Los Cardones tiene una gran importancia por la conformación física de sus ambientes y por su finalidad que es conservar un sector con biomas andinos representativos de la Puna y el Monte argentino (SIB-APN, 2012).

Muestreo: Los artrópodos tienen una mayor actividad en estos ambientes de altura durante el otoño y el verano, estaciones en las cuales se realizó un muestreo en Mayo 2007 y otro en Febrero 2008. En cada ecorregión, PAN (Valle Encantado), MO (Quebrada de Cajoncillos) y PP (Recta de Tin-Tin), se seleccionaron tres sitios de muestreo separados entre sí por lo menos por un kilómetro (Fig. 1). En cada uno de ellos se tomaron cinco muestras con trampas de caída (pit-fall) para artrópodos de suelo, y cinco con un G-Vac (garden-vaccum) (Bell, Wheater, Henderson \& Cullen, 2000) sobre la vegetación en los lugares donde existía, y alejadas a una distancia mínima de $10 \mathrm{~m}$ de las trampas de caída.
Se tomaron las muestras de artrópodos sobre la vegetación comenzando el primer sitio a las 10AM y el último a las 3PM. Una muestra estuvo representada por la succión de la vegetación en un área de un metro cuadrado por el término de un minuto. Las trampas de caída, con solución salina como conservante (sal agua $(\mathrm{p} / \mathrm{v})$ en proporción $1: 8$, con gotas de detergente), estuvieron dispuestas a lo largo de una transecta lineal, separadas entre sí por $10 \mathrm{~m}$ y con actividad de 10 días, que al finalizar se recolectaron los artrópodos capturados. Se consideraron ambos métodos de muestreo por ser complementarios (Borges \& Brown, 2003). Se tomaron 90 muestras por cada estación del año (nueve sitios $\mathrm{X}$ dos técnicas de recolecta $\mathrm{X}$ cinco muestras), las que fueron debidamente rotuladas y consideradas como independientes. El material de artrópodos fue separado en órdenes y familias por medio de claves taxonómicas disponibles (Borror, Triplehorn \& Johnson ,1989; De Santis, 1969; Ramírez, 1999), y en especies/morfoespecies. Adicionalmente, se generó una base de datos de fotos digitalizadas de cada una de ellas y sus caracteres distintivos por medio del programa TAXIS ver.3.5 (Meyke, 1999-2004). El material recolectado se encuentra depositado en la Colección IEBIMuseo de Ciencias Naturales de la Universidad Nacional de Salta.

Para el inventario y diversidad alfa: $\mathrm{Se}$ generaron curvas de acumulación de especies basadas en individuos para todo el muestreo del parque y por ecorregión por medio del programa EstimateS 7.0 (Colwell, 2004). Se comparó los valores de riqueza de especies observadas con respecto a la esperada por medio de estimadores no paramétricos, considerando Michaelis-Menten, porque produce estimaciones estables y ampliamente precisas en un número pequeño de muestras (Magurran, 2004), sumado a Chaol y Jacknife1 porque dan buenas resoluciones (Walther \& Moore, 2005). Con ellos se confirmó el nivel de completitud del inventario obtenido. Para comparar la diversidad entre las ecorregiones, se generaron los "perfiles de diversidad" usando la familia de índices de diversidad uniparamétrica de 


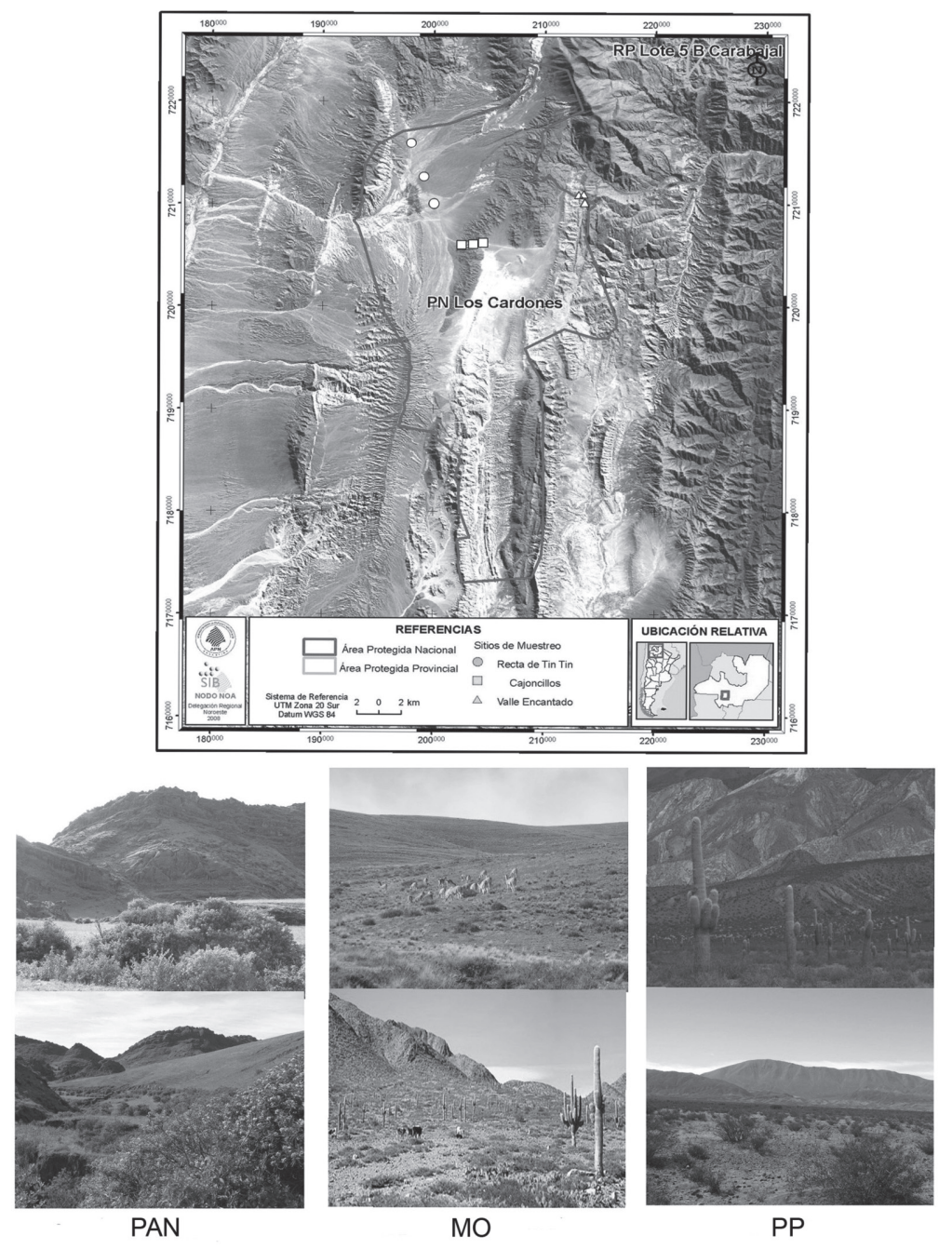

Fig. 1. Localización de los sitios de muestreo en el Parque Nacional Los Cardones, Salta (Argentina). PAN: Pastizal Altoandino Nublado de las Yungas, MO: Monte Occidental, PP: Prepuna-Puna.

Fig. 1. Location of sampling sites in the Parque Nacional Los Cardones, Salta (Argentina). PAN: High Andean Grassland of the Yungas, MO: Occidental Monte, PP: Prepuna-Puna.

Rényi (Tóthmérész, 1998) con el programa PAST ver. 2.14 (Hammer, Harper \& Ryan, 2003), que permite una comparación escalable de la diversidad de los ensambles de dos o más comunidades (Moreno, Barragán, Pineda \& Pavón, 2011). También se estimó la "verdadera diversidad" (Jost 2006, 2007) por medio de los "números efectivos de especies" utilizando el software SPADE (Chao \& Shen, 2003-2005). Se consideró como la riqueza total de especies (verdadera diversidad de orden cero) el valor del estimador ACE (Abundance-based coverage estimator) para comunidades altamente heterogéneas $\left(\mathrm{CV}_{\text {-rare }}>0.8\right)$ (Chao \& Shen, 2003); el exponencial del índice de Shannon (la verdadera diversidad de orden 1), y el inverso del índice de Simpson (la verdadera diversidad de orden 2) calculado usando el estimador MVUE (Minimum variance unbiased estimator) (Moreno et al., 2011). 
Diversidad beta: Con el mismo programa se calculó la disimilitud (o recambio de especies) de los ensambles de artrópodos registrados en los diferentes sitios y ecorregiones por medio del índice de disimilitud de Morisita. La complementariedad de los inventarios obtenidos entre ecorregiones fue analizado usando el índice de Vane-Wright, Humphries \& Williams (1991). Se realizó un ordenamiento de los sitios muestreados por medio de un análisis de escalamiento no métrico multidimensional (NMDS) usando el programa PC-ORD vers.6 (McCune \& Mefford, 2011) siguiendo la rutina propuesta por Peck (2010): primero corriendo con el comando "Autopilotslow-thorough", usando Sorensen como medida de distancia (500 iteraciones), configuración de inicio al azar, con 250 corridas con datos reales y una corrida reduciendo la dimensión para cada ciclo. El valor del stress de acuerdo con el PCORD proviene de multiplicar el valor obtenido por 100, por lo que se expresa en porcentaje (McCune \& Mefford, 2002). Una vez obtenido el ordenamiento de los sitios, los valores de similitud entre los diferentes grupos resultantes fueron sometidos a un procedimiento de permutación múltiple (MRPP) para evaluar si existieron diferencias significativas entre los valores de similitud obtenidos entre ellos. Además, se compararon los valores de abundancias y riqueza de especies de las familias de los órdenes más diversos registrados en cada ecorregión por medio de un análisis de porcentaje de similitud (SIMPER), teniendo en cuenta la disimilitud de Bray-Curtis, calculado por medio del programa PAST ver. 2.14 (Hammer et al., 2003), para determinar qué familias de estos grupos contribuyeron más a la disimilitud entre las ecorregiones.

\section{RESULTADOS}

Inventario y diversidad: Un total de 10637 artrópodos fueron recolectados y correspondieron a 469 especies/morfoespecies de 13 órdenes. Los ambientes de la ecorregión Yungas (PAN) mostraron una mayor abundancia y riqueza de especies en comparación con las otras ecorregiones, siendo la Prepuna-Puna la menos rica en especies (Fig. 2). La curva de acumulación de especies por individuos para el

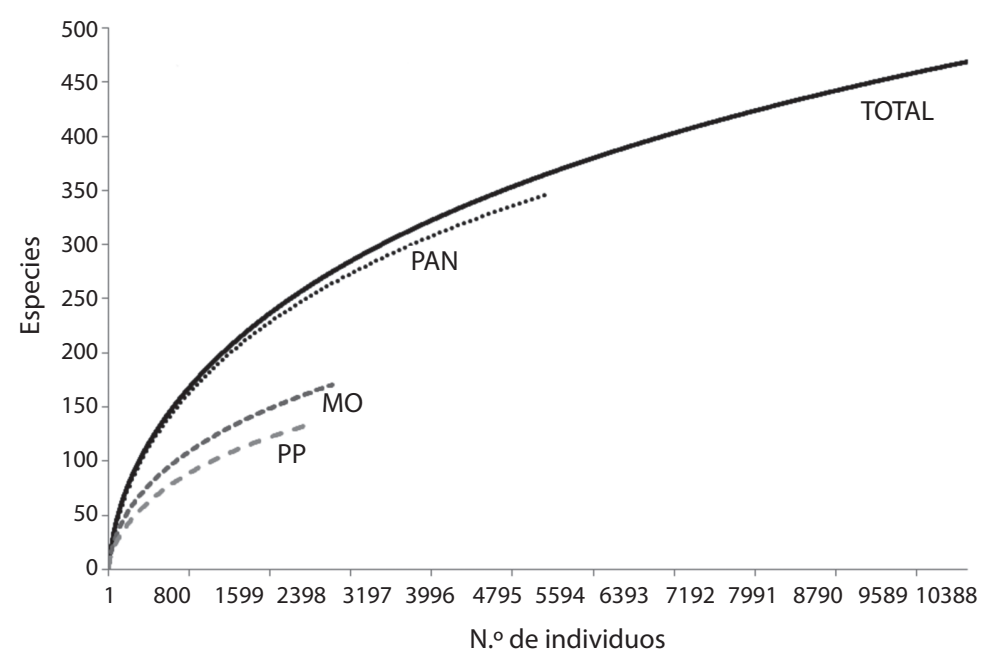

Fig. 2. Curva de acumulación de especies por individuos de todo el muestreo (TOTAL) y por cada ecorregión del Parque Nacional Los Cardones (2007-2008). PAN: Pastizal Altoandino Nublado de las Yungas, MO: Monte Occidental, PP: Prepuna-Puna.

Fig. 2. Species accumulation curve base don individuals considering the complete inventory (TOTAL), and by eco-region in the Parque Nacional Los Cardones (2007-2008). PAN: High Andean Grassland of the Yungas, MO: Occidental Monte, PP: Prepuna-Puna. 
muestreo total no llegó a una asíntota debido a la alta heterogeneidad ambiental, sin embargo, se pudo comprobar que el inventario obtenido fue bueno alcanzando casi un $81 \%$ de lo estimado por el estimador más próximo (Michaelis-Menten) a la riqueza de especies observada (Cuadro 1). Los inventarios obtenidos en cada ecorregión superaron en todos los casos el 65\% de los valores esperados por el estimador más próximo, evidenciándose que el inventario del PAN fue el más completo donde MichaelisMenten y Chao1 calcularon un 26.18 y $28.58 \%$ de especies más que las observadas, respectivamente (Cuadro 1).

La diversidad de las ecorregiones comparada por medio de los perfiles de diversidad mostró que los sitios de la ecorregión Yungas (PAN) fueron los más diversos (Fig. 3), siendo

\section{CUADRO 1}

Riqueza de especies y abundancia total por ecorregión y los valores de los estimadores no paramétricos de riqueza más próximos a los valores del Sobs, en el PN Los Cardones, Salta (2007-2008)

TABLE 1

Species richness and total abundance by eco-region, including the values of non-parametric estimators more closer to the observed species richness value (Sobs), in de PN Los Cardones (2007-2008)

\begin{tabular}{|c|c|c|c|c|}
\hline & Abundancia & Riqueza de especies & Riqueza de especies estimada & Completitud del inventario (\%) \\
\hline \multirow[t]{3}{*}{ TOTAL } & 10.637 & 469 & $\mathrm{MM}=581.98$ & 80.59 \\
\hline & & & Chao $1=664.36 \pm 41.15$ & 70.59 \\
\hline & & & Jack1=663.91 \pm 19.91 & 70.64 \\
\hline \multirow[t]{3}{*}{ PAN } & 5.438 & 347 & $\mathrm{MM}=470.08$ & 73.82 \\
\hline & & & Chao $1=485.85 \pm 32.68$ & 71.42 \\
\hline & & & Jack $1=500.4 \pm 16.12$ & 69.34 \\
\hline \multirow[t]{3}{*}{ MO } & 2.771 & 171 & $\mathrm{MM}=243.08$ & 70.35 \\
\hline & & & Chao $1=285.57 \pm 38.09$ & 59.88 \\
\hline & & & Jack $1=257.53 \pm 9.66$ & 66.4 \\
\hline \multirow[t]{3}{*}{ PP } & 2.428 & 132 & $\mathrm{MM}=182.43$ & 72.36 \\
\hline & & & Chaol $=193.07 \pm 22.46$ & 68.37 \\
\hline & & & Jack $1=197.88 \pm 9.31$ & 66.71 \\
\hline
\end{tabular}

PAN: Pastizal Altoandino Nublado de las Yungas, MO: Monte Occidental, PP: Prepuna-Puna.

PAN: High Andean Grassland of the Yungas, MO: Occidental Monte, PP: Prepuna-Puna.

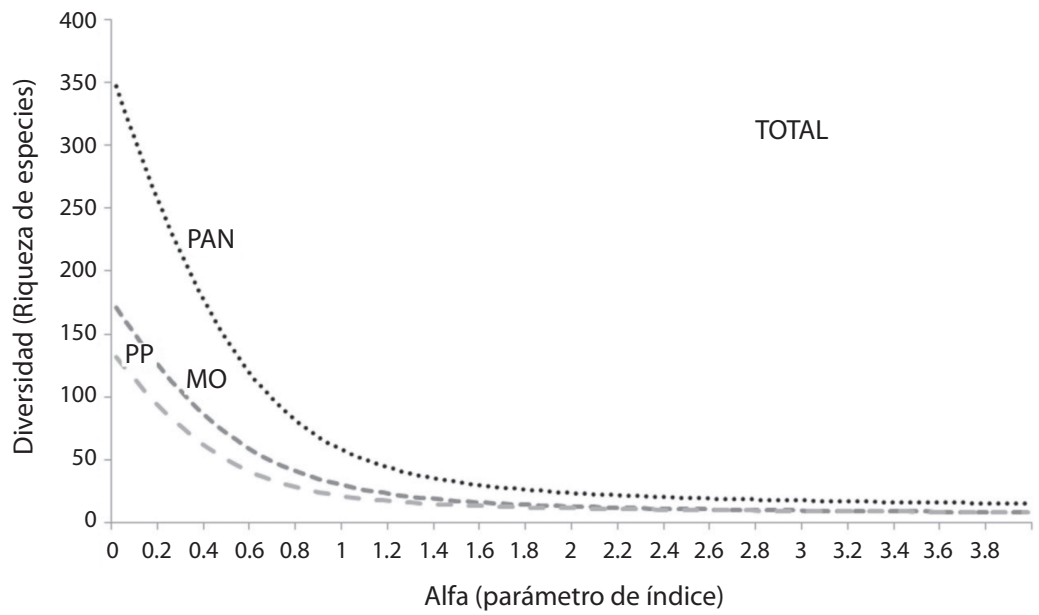

Fig. 3. Perfiles de diversidad de los artrópodos en las ecorregiones del Parque Nacional Los Cardones (2007-2008). PAN: Pastizal Altoandino Nublado de las Yungas, MO: Monte Occidental, PP: Prepuna-Puna.

Fig. 3. Diversity profiles of arthropods by eco-regions of PN Los Cardones (2007-2008). PAN: High Andean Grassland of the Yungas, MO: Occidental Monte, PP: Prepuna-Puna. 
CUADRO 2

"Verdadera diversidad" considerando todo el muestreo y en cada ecorregión, mostrando los valores de los números efectivos de diversidad observada y estimada, y el porcentaje del completitud de inventario tomando en cuenta los valores de Sobs y ACE (Abundance-based Coverage Estimator)

TABLE 2

"True diversity" considering all the inventory and by eco-regions, showing the values of the effective number of diversity observed and estimated, and the percentage of the inventory completion taking in account the Sobs and the ACE value (Abundance-based Coverage Estimator)

\begin{tabular}{lccccccc}
$r$ & \multicolumn{3}{c}{ Diversidad Observada } & \multicolumn{3}{c}{ Diversidad Estimada } & $\begin{array}{c}\text { Completitud } \\
\text { de inventario }\end{array}$ \\
& ${ }^{0} \mathrm{D}$ & ${ }^{1} \mathrm{D}$ & ${ }^{2} \mathrm{D}$ & ${ }^{0} \mathrm{D}_{\text {(ACE) }}$ & ${ }^{1} \mathrm{D}_{\text {(MLE-bc) }}$ & ${ }^{2} \mathrm{D}_{\text {(MVUE) }}$ & $71.57 \%$ \\
TOTAL & 469 & 68.7 & 30.99 & $655.3 \pm 30.70$ & $70.8 \pm 8.41$ & $31.1 \pm 0.12$ & $68.62 \%$ \\
PAN & 347 & 56.06 & 23.33 & $505.7 \pm 29.10$ & $58.7 \pm 8.85$ & $23.4 \pm 0.14$ & $63.15 \%$ \\
MO & 171 & 28.59 & 12.36 & $270.8 \pm 25.9$ & $30.0 \pm 6.42$ & $12.4 \pm 0.21$ & $63.58 \%$ \\
PP & 132 & 20.28 & 10.79 & $207.6 \pm 21.50$ & $21.2 \pm 3.44$ & $10.8 \pm 0.23$ & \\
\hline
\end{tabular}

Diversidad observada y estimada: ${ }^{0} \mathrm{D},{ }^{1} \mathrm{D}$ y ${ }^{2} \mathrm{D}$ (denotan medidas de diversidad de orden 0,1 y 2 , respectivamente). Diversidad observada (usando PAST software): ${ }^{0} \mathrm{D}=\mathrm{S}_{\text {obs }}$ (riqueza de especies observada); ${ }^{1} \mathrm{D}=$ exponencial del índice de Shannon; ${ }^{2} \mathrm{D}=$ inversa del índice de Simpson. Diversidad estimada (usando SPADE software): ${ }^{0} \mathrm{D}=\mathrm{ACE}$ (Abundance-based Coverage Estimator); ${ }^{1} \mathrm{D}=$ bias-controled índice de Shannon; ${ }^{2} \mathrm{D}=\mathrm{MVUE}$ (mínimum variance unbiased estimator). PAN: Pastizal Altoandino Nublado de las Yungas, MO: Monte Occidental, PP: Prepuna-Puna.

Observed and estimated diversity: $\mathrm{D}, \mathrm{D}$ and $\mathrm{D}$ (denote diversity measures of order 0,1 and 2, respectively). Observed diversity (using PAST software): $0 \mathrm{D}=$ Sobs (observed species richness); ${ }^{1} \mathrm{D}=$ exponential of Shannon Index; ${ }^{2} \mathrm{D}=$ inverse of Simpson index. Estimated diversity (using SPADE software): ${ }^{0} \mathrm{D}=\mathrm{ACE}$ (Abundance-based Coverage Estimator); $\mathrm{D}=$ biascontroled Shannon index; ${ }^{2} \mathrm{D}=\mathrm{MVUE}$ (minimum variance unbiased estimator). PAN: High Andean Grassland of the Yungas, MO: Occidental Monte, PP: Prepuna-Puna.

1.96 veces mayor que la comunidad de artrópodos del MO, y 2.76 veces mayor que la de la PP (Cuadro 2), al comparar los valores de ${ }^{1} \mathrm{D}$ observada (diversidad de orden 1). Además, la completitud del inventario fue buena, si se compara los valores de los números efectivos de la riqueza observada con la estimada (Cuadro 2), mostrando en todos los casos un porcentaje de completitud de inventario mayor al 63\%.

En el ordenamiento resultante del NMDS se pudo reconocer tres grupos correspondientes a las ecorregiones representadas en el Parque Nacional Los Cardones (Fig. 4). El ordenamiento en dos ejes explicó el $80.40 \%$ de la varianza (Eje $1=0.618$, Eje $2=0.186$ ) y con un Stress $=11.52$, explicando el primer eje la existencia de diferentes comunidades de artrópodos en cada una de las ecorregiones. Esto fue comprobado en el MRPP que resultó en una diferencia altamente significativa entre las comunidades de artrópodos analizadas $(\mathrm{A}=0.5999, \mathrm{p}=0.00071)$. Por ello, las comunidades de artrópodos de las ecorregiones del
Parque Nacional Los Cardones comparten muy pocas especies entre ellas, pues sus ensambles mostraron un elevado valor de disimilitud $\left(1-\mathrm{C}_{33}=0.853\right)$, llegando hasta valores de complementariedad del $85 \%$ entre las comunidades del PAN y la PP (Cuadro 3). Los sitios de una misma ecorregión mostraron ensambles más similares entre sí que con otros sitios de otras ecorregiones. A pesar de ello, se registró un recambio de especies en los sitios de una misma ecorregión, donde los valores de similitud variaron entre el $35 \%\left(1-\mathrm{C}_{33}=0.653\right)$ entre los sitios del PAN, hasta valores del $52 \%$ $\left(1-\mathrm{C}_{33}=0.478\right)$ en los de la PP; mientras que el $43 \%\left(1-\mathrm{C}_{33}=0.569\right)$ se observó entre los sitios del MO.

Ensambles de artrópodos y sus variaciones: Las arañas, dípteros e himenópteros fueron los grupos de artrópodos más diversos en todas las ecorregiones (Fig. 5). Entre las arañas, de acuerdo con el análisis SIMPER, las familias que más aportaron a la disimilitud 


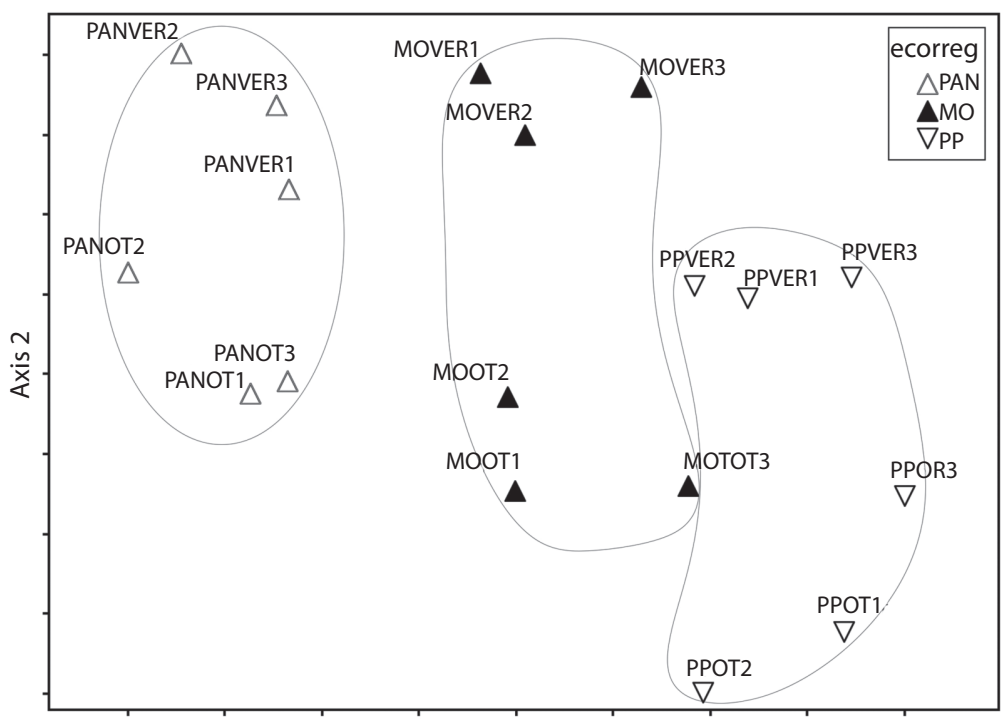

Axis 1

Fig. 4. Ordenamiento por medio de un análisis de escalamiento multidimensional no métrico (NMDS) de los sitios muestreados en el Parque Nacional Los Cardones (2007-2008), mostrando diferencias en la fauna de artrópodos por ecorregión del parque. (Eje1=0.618, Eje2=0.186) y con un Stress=11.52.PAN: Pastizal Altoandino Nublado de las Yungas, MO: Monte Occidental, PP: Prepuna-Puna; OT: otoño, VER: verano; 1, 2, 3: sitios uno, dos y tres, respectivamente.

Fig. 4. Ordination by NMDS analysis (non-metric multidimentional scaling) of the sampling sites of the Parque Nacional Los Cardones (2007-2008), showing differences between eco-regional fauna in the park. (Exe 1=0.618, Exe 2=0.186), Stress=11.52 PAN: High Andean Grassland of the Yungas, MO: Occidental Monte, PP: Prepuna-Puna; OT: autumn, VER: summer; 1, 2 and 3: sites one, two and three, respectively.

\section{CUADRO 3}

Porcentaje de complementariedad entre ecorregiones del PN Los Cardones, Salta (2007-2008) según el índice de complementariedad de Vane Wright et al. (1991)

TABLE 3

Complementary percentage between eco-regions of the Parque Nacional Los Cardones, Salta (2007-2008), considering the complementarity index of Vane Wright et al. (1991)

\begin{tabular}{lccc} 
& PAN & MO & PP \\
PAN & - & $(95)$ & $(62)$ \\
MO & 77.5 & - & $(68)$ \\
PP & 85.13 & 71.06 & - \\
\hline
\end{tabular}

Entre paréntesis se presenta el número de especies compartidas. PAN: Pastizal Altoandino Nublado de las Yungas, MO: Monte Occidental, PP: Prepuna-Puna.

The number of shared species is shown between parenthesis. PAN: High Andean Grassland of the Yungas, MO: Occidental Monte, PP: Prepuna-Puna. entre las comunidades ecorregionales fueron Salticidae y Dyctinidae, sumado a Anyphaenidae que fue la familia dominante en el PAN. Sciaridae, Tephritidae y Muscidae fueron los dípteros que más contribuyeron a la disimilitud entre las ecorregiones, mientras que Chironomidae fue la más abundante en el PAN y no se registró en la PP. En cuanto a los himenópteros, Formicidae fue la familia dominante y la que presentó el mayor número de especies en las tres ecorregiones, mostrando cambios en los ensambles entre ellas. El mayor recambio de especies de hormigas entre ecorregiones fueron registradas para las especies de Camponotus, Brachymyrmex y Pheidole.

Al comparar la abundancia y la riqueza de especies de artrópodos entre estaciones, se evidenció que la diversidad fue mayor en el verano, obteniéndose resultados similares al comparar 


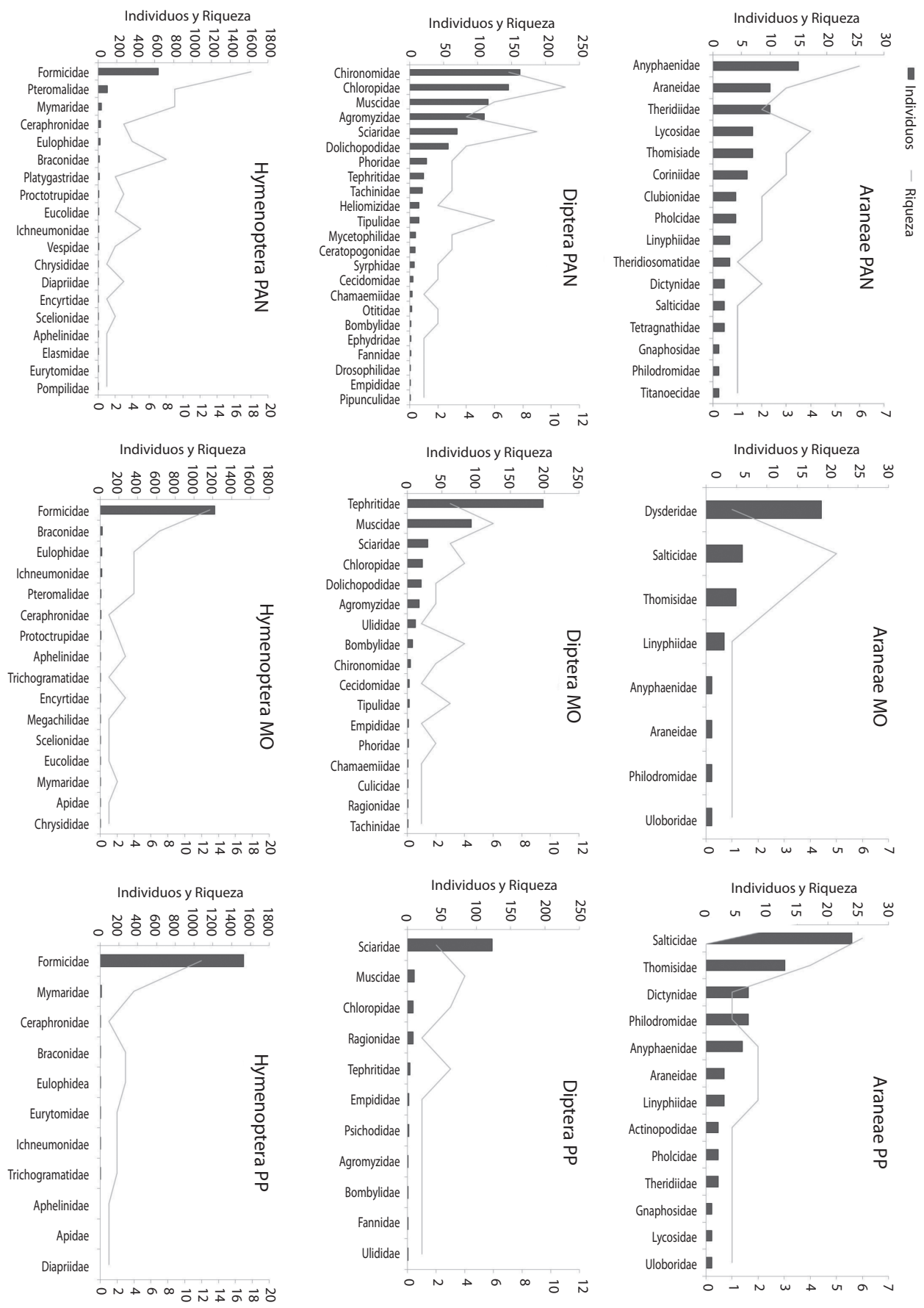

Fig. 5. Representación de la riqueza de especies y abundancia por familias de los órdenes de artrópodos más diversos registrados en las ecorregiones del Parque Nacional Los Cardones (2007-2008). PAN: Pastizal Altoandino Nublado de las Yungas, MO: Monte Occidental, PP: Prepuna-Puna.

Fig. 5. Species richness and abundances by families of the most diverse arthropod orders recorded by eco-regions in the Parque Nacional Los Cardones (2007-2008).PAN: High Andean Grassland of the Yungas, MO: Occidental Monte, PP: Prepuna-Puna. 


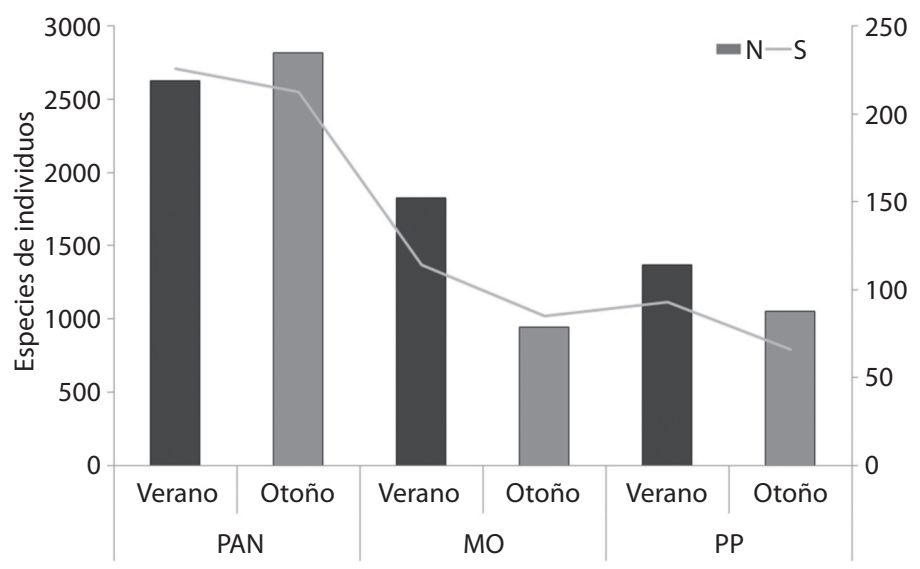

Fig. 6. Variación estacional de la abundancia y riqueza de especies de artrópodos en diferentes ecorregiones del Parque Nacional Los Cardones (2007-2008).PAN: Pastizal Altoandino Nublado de las Yungas, MO: Monte Occidental, PP: Prepuna-Puna. N: número de individuos, S: riqueza de especies.

Fig. 6. Seasonal variation of the species richness and abundance of arthropods collected in different eco-regions of the Parque Nacional Los Cardones (2007-2008). PAN: High Andean Grassland of the Yungas, MO: Occidental Monte, PP: Prepuna-Puna. N: number of individuals, S: species richness.

entre estaciones y ecorregiones (Fig. 6). Los patrones de riqueza de especies fueron similares entre las dos estaciones, pero la abundancia mostró un incremento en los sitios de PAN en el otoño con respecto al verano.

\section{DISCUSIÓN}

Este estudio al ser el primero en analizar las comunidades de artrópodos en el Parque Nacional Los Cardones no sólo contribuye al conocimiento de la diversidad existente en esta área natural protegida, sino que permitirá hacer futuras comparaciones con otras áreas del parque $\mathrm{u}$ otros ambientes de las mismas ecorregiones dentro y fuera del mismo. De acuerdo con los resultados obtenidos, este parque nacional está conservando y protegiendo, al mantener sin alteración estos ambientes de altura, una importante y diversa comunidad de artrópodos que estaría representada por más de 520 especies, las 469 registradas en este estudio sumadas a los registros de lepidópteros (Moschione, 2009), que en nuestros muestreos no obtuvieron buenos registros por las técnicas de recolecta utilizadas. El inventario total obtenido es bueno superando el $80 \%$ de las especies estimadas por los estimadores no paramétricos. Iguales resultados se obtienen para las diferentes ecorregiones muestreadas donde los valores de completitud de inventario superaron el 70\% con respecto al valor de Michaelis-Menten. Los ambientes de Yungas (PAN) son los más diversos del parque y los menos diversos los de Prepuna-Puna (PP), posiblemente relacionado con las diferencias observadas en la vegetación en estos ambientes.

Cada ecorregión presente en el parque posee una comunidad de artrópodos particular, debido a que sólo un $15 \%$ de las especies de artrópodos registrados en este estudio son comunes a las tres ecorregiones del parque. Ello se demuestra en el ordenamiento obtenido por el NMDS y las diferencias altamente significativas resultantes del MRPP entre las comunidades producto del ordenamiento. Estos resultados soportarían la idea de Orians (1993), que las ecorregiones funcionan como unidades de conservación a gran escala. Las ecorregiones al ser unidades relativamente grandes de tierra y agua que contienen un conjunto de comunidades naturales características comparten una gran cantidad de sus especies, una dinámica ecológica y condiciones ambientales 
(Dinerstein et al., 1995; Groves et al., 2000). Por otro lado, los sitios de una misma ecorregión muestran mayores valores de similitud en sus ensambles de artrópodos que con los de otras ecorregiones, a pesar de existir un recambio de especies. Estos resultados son consistentes con los obtenidos por Paknia \& Pfeiffer (2011), quienes mencionan que la diversidad beta entre sitios locales de una ecorregión contribuyen de manera significativa a la diversidad de la ecorregión, por lo que este recambio de especies entre sitios debe ser tenido en cuenta en planes de conservación.

Las diferencias observadas en la estructura de la vegetación entre las ecorregiones del Parque Nacional Los Cardones podrían ser uno de los factores que determinaría la alta variación en la abundancia y riqueza de artrópodos registrada. Por su parte, el Pastizal Altoandino Nublado, que posee una estructura de la vegetación más compleja, sumado a una mayor cobertura vegetal sobre el suelo que la Prepuna-Puna y el Monte Occidental, muestra una mayor riqueza de especies y abundancia de artrópodos, que nos lleva a pensar que el PAN debe contener una mayor cantidad de microhábitats que están siendo ocupados por una comunidad de artrópodos más diversa. Esto se apoya en la idea que varios grupos de artrópodos se asocian positivamente con la complejidad del hábitat (Gardner, Cabido, Valladares \& Diaz, 1995; Humphrey, Pearce, Ferris-Khan \& Jukes, 1999; Hansen, 2000).

En el caso de los ambientes áridos, diferentes grupos de especies desarrollan estrategias ecológicas específicas para obtener mayor provecho de los recursos disponibles en la fase húmeda (Cepeda-Pizarro, Pizarro-Araya \& Vásquez, 2005). Esto queda reflejado en los resultados aquí obtenidos, ya que en el parque la diversidad de artrópodos es mayor en el verano, lo que podría explicarse por un cambio estacional de la vegetación. En el caso de los ambientes más xerófilos del parque, como lo son el MO y la PP, los recursos vegetales son máximos en los períodos lluviosos que corresponden al período estival. En contraposición, el incremento de la abundancia de los artrópodos en el PAN durante el otoño podría deberse a que el recurso vegetal allí se mantiene por mayor tiempo, a causa de la existencia de una mayor humedad en el suelo y en el ambiente llegando a precipitaciones medias anuales en el orden de los $600 \mathrm{~mm}$ en el Valle Encantado (Bianchi, Neumann, Noé \& González et al., 2006), las mayores de todo el parque.

Por otro lado, las variaciones observadas en la riqueza, abundancia y composición de ensambles en la comunidad de artrópodos en cada ecorregión y entre estaciones, no sólo podrían explicarse por los cambios estacionales propios de la vegetación, sino también a las características cuantitativas y cualitativas del componente edáfico (Sánchez \& AmatGarcía, 2005). Éste es un factor importante que también podría estar contribuyendo a la variabilidad observada entre las comunidades estudiadas. La cobertura vegetal sobre el suelo es mayor en los ambientes del PAN, a lo que se le suma una mayor cantidad de materia orgánica muerta sobre él en el otoño, lo que se reflejó en un incremento de la abundancia de dos especies de ácaros del suelo en esa estación. Por otro lado, las hormigas son dominantes en los períodos lluviosos en el Monte Occidental y la Prepuna-Puna donde hay mayor disponibilidad de recursos vegetales.

Algunos grupos de artrópodos fueron dominantes con respecto a otros en los distintos ambientes del Parque Nacional Los Cardones, especialmente las arañas, los dípteros y los formícidos. Las arañas son un grupo clave en cualquier ecosistema, y pueden ser utilizadas como indicadoras de calidad ambiental (Pinkus-Rendón, León-Cortés \& Ibarra-Nuñez, 2006) ya que son dependientes del hábitat y su complejidad (Weeks \& Holtzer, 2000; Pearce, Venier, Eccles, Pedlar \& McKenney, 2003). Por ello, en nuestro estudio son abundantes y diversas en ambientes del PAN del parque. En cambio, su diversidad y abundancia es baja en ambientes del Monte Occidental, explicable porque esta ecorregión sería un Chaco empobrecido (Stange, Terán \& Willink, 1976), y la diversidad de artrópodos es menor que en aquella ecorregión, aunque puede mostrar valores 
altos de endemismos (Roig-Juñent, Flores, Claver, Debandi \& Marvaldi, 2001).

En el caso de los dípteros, ellos también han sido propuestos como un grupo indicador de biodiversidad y del estado de los ecosistema (Hanski \& Koskela, 1977), ya que sus patrones de diversidad están estrechamente relacionados con el tipo de hábitat y la disponibilidad de alimento (Woodcock, Watt \& Leather, 2003); al igual que las hormigas que muestran ser buenas indicadoras ecológicas y/o de biodiversidad (Arcila \& Lozano-Zambrano, 2003). Debido a ello, los dípteros son más diversos y abundantes también en los ambientes del Pastizal Altoandino Nublado de las Yungas del parque, lo que es compartido por las hormigas que muestran allí una mayor riqueza de especies, aunque son más abundantes en ambientes del MO y PP. Las comunidades de hormigas son pobres en los matorrales y los pastizales áridos (Flores-Maldonado, Phillips \& SánchezRamos, 1999; Rojas \& Fragoso, 2000), posiblemente ello explique la menor riqueza de especies registradas en ambientes del Monte Occidental y la Prepuna-Puna del parque. La mayor abundancia registrada en estos últimos ambientes durante el otoño podría indicar que ellas utilizan los recursos disponibles en los cortos períodos en que se encuentran accesibles, principalmente después de las lluvias.

Concluyendo, existe una alta diversidad alfa en cada ambiente y una elevada diversidad beta entre las ecorregiones del Parque Nacional Los Cardones que contribuye a que el parque esté conservando una considerable diversidad de artrópodos de la región. Por otro lado, los diferentes ambientes de una misma ecorregión también están contribuyendo en el mantenimiento de la diversidad regional de artrópodos del parque. Allí, se pudo reconocer que tres grupos de artrópodos son muy diversos y abundantes. Ellos son reconocidos como grupos indicadores de hábitat y de diversidad, por lo que podrían ser utilizados en futuros estudios como grupos focales para evaluar la diversidad de artrópodos y sus cambios en estos y otros ambientes del parque, como así también en las ecorregiones aquí analizadas.

\section{AGRADECIMIENTOS}

A la Administración de Parques Nacionales por permitirnos el acceso al Parque Nacional Los Cardones; a CIUNSa (Consejo de Investigaciones de la Universidad Nacional de Salta) y CONICET (Consejo Nacional de Investigaciones Científicas y Técnicas) por su apoyo.

\section{RESUMEN}

El Parque Nacional Los Cardones ubicado en la provincia de Salta protege las ecorregiones de Puna, Monte y Pastizal Altoandino Nublado de la Yungas. Nuestro objetivo es evaluar la diversidad alfa y beta de artrópodos en las ecorregiones del Parque. Se muestrearon tres sitios en cada ecorregión utilizando Pit-fall y G-Vac. Se calcularon estimadores no paramétricos de riqueza de especies, la diversidad entre ecorregiones se comparó a través de los "perfiles de diversidad" y "números efectivos de especies", se analizó el recambio de especies y la complementariedad entre las ecorregiones y sitios de una misma ecorregión. Se registraron $469 \mathrm{spp} /$ morfoespecies, la diversidad en el Parque fue alta, siendo mayor en los Pastizal Altoandino Nublado, y la más baja en la Puna. El inventario llegó al $81 \%$ de completitud. El recambio de especies entre ecorregiones fue alto, de esa manera los diferentes ambientes del Parque contribuyen a conservar la diversidad regional de artrópodos. Las ensambles de artrópodos en sitios de una misma ecorregión mostraron mayor similitud entre sí que con sitios más distantes. Se pudo reconocer tres grupos de artrópodos (arañas, dípteros e himenópteros) que son diversos y abundantes, los que podrían ser utilizados en futuros estudios como grupos focales.

Palabras claves: artrópodos, diversidad alfa, diversidad beta, ecorregiones, área natural protegida, Argentina.

\section{REFERENCIAS}

Arcila, A. M. \& Lozano-Zambrano, F. H. (2003). Hormigas como herramientas para la bioindicación y el monitoreo, p. 159-166. In F. Fernández (Ed.). Introducción a las hormigas de la región neotropical. Bogotá, Colombia: IAvH.

Bell, J. R., Wheater, C. P., Henderson, R. \& Cullen, W. R. (2000). Testing the efficiency of suction samplers (G-vacs) on spiders: the effect of increasing nozzle size and suction time. European Arachnology, 285-290.

Bianchi, A. R., J. N. Volante, R. Neumann, Y. Noé \& A. C. González. (2006). Mapa digital de isohietas anuales del NOA. (Consulado: 01 Junio 2012, http://www. 
inta.gov.ar/prorenoa/info/resultados/Isohietas/isohietas_anuales.htm).

Borges, P. A. V. \& Brown, V. K. (2003). Estimating species richness of arthropods in Azorean pastures: the adequacy of suction sampling and pitfall trapping. Graellsia, 59, 7-24.

Borror, D. J, Triplehorn, C. A. \& Johnson, N. F. (1989). An introduction to the study of insects. Harcout Brace. Orlando, USA: Sunders College Publishing.

Brown, A., Martínez-Ortiz, U., Acerbi, M. \& Concuera, J. (2006). Situación Ambiental Argentina 2005. Buenos Aires: Fundación Vida Silvestre Argentina.

Cardoso, P., Borges, P. A. V., Dinis, F. \& Gaspar, C. (2010) Patterns of alpha and beta diversity of epigean arthropods at contrasting land-uses of an oceanic island (Terceira, Azores), p. 73-88. In A.R.M. Serrano, P.A.V. Borges, M. Boieiro \& P. Oromí (Eds.), Terrestrial arthropods of Macaronesia. Biodiversity, Ecology and Evolution. Lisboa: Sociedad Portuguesa de Entomología.

Cava, M. B., Corronca, J. A. \& Coscarón, M. C. (2012). Composition and structure of Heteroptera communities (Hemiptera) in high altitude habitats of a National Protected area in Argentina. Munis Entomology \& Zoology, 7, 1214-1225.

Cepeda-Pizarro, J., Pizarro-Araya, J. \& Vásquez, H. (2005). Composición y abundancia de artrópodos epigeos del Parque Nacional Llanos de Challe: impactos del ENOS de 1997 y efectos del hábitat pedológico. Revista Chilena de Historia Natural, 78, 635-650.

Chao, A. \&. Shen, T. (2003). Nonparametric estimation of Shannon's index of diversity when there are unseen species in sample. Environmental and Ecological Statistics, 10, 429-443.

Chao, A. \& T. Shen. (2003-2005). Program SPADE. Species Prediction and Diversity Estimation. (Consultado 01 Julio 2012, http://chao.stat.nthu.edu.tw).

Chapin, F. S., Walker, B. H., Hobbs, R. J., Hopper, D. U., Lawton, J. H. \& Sala, O. E. (1997). Biotic control over the functioning of ecosystems. Science, 277, 500-504.

Chebez, J. C. (2005). Noroeste. Guía de las reservas Naturales de la Argentina. Buenos Aires: Editorial Albatros.

Colwell, R. (2004). EstimateS 7.0b. Statistical estimation of species richness and shared species from simples (Recuperado de http://vivceroy.eeb.uconn.edu).

De Santis, L. (1969). Hymenoptera: clave de las familias con representantes entomófagos. Serie didáctica $\mathrm{N}^{\circ} 6$. Facultad de Agronomía y Zootecnia, Universidad Nacional de Tucumán.

Dinerstein, E., Olson, D. M., Graham, D. J., Webster, A. L., Primm, S. A., Bookbinder, M. P \& Ledec, G. (1995). Una evaluación del estado de conservación de las ecorregiones terrestres de América Latina y el Caribe. Washington, District of Columbia, USA: World Wildlife Foundation and World Bank.

Flores-Maldonado, K. Y., Phillips, S. A. \& SánchezRamos. G. (1999). The myrmecofauna (Hymenoptera: Formicidae) along an altitudinal gradient in the Sierra Madre Oriental of Northeastern Mexico. Southwestern Naturalist, 44, 457-461.

Gardner, S. M., Cabido, M. R., Valladares, G. R. \& Diaz, S. (1995). The influence of habitat structure on arthropod diversity in Argentine semi-arid Chaco forest. Journal of Vegetation Science, 6, 349-356.

Gonzales del Solar, R. S., Puig, J., Videla, F. \& Roig, V. (1997). Diet composition of the South American grey fox, Pseudalopex griseus Gray, 1837 in Northeasterm Mendoza, Argentina. Mammalia, 61, 617-621.

Gray, C. \& Jongepier, E. (2008). Effect of human disturbance on arthropod diversity at Kirindy Forest, Western Madagascar. Tropical Biology Assessment, 9-10.

Groves, C., Valutis, L., Vosick, D., Neely, B., Wheaton, K., Touval, J. \& Runnels, B. (2000). Designing a Geography of Hope: A practitioner's handbook to ecoregional conservation planning. Arlington, VA: The Nature Conservancy.

Hammer, O., D. A. T. Harper \& P. D. Ryan. (2003). PAST (Paleontological Statistics) version. 1.18. (Recuperado http://folk.uio.no/ohammer/past/)

Hansen, R. A. (2000). Effects of habitat complexity and composition on a diverse litter microarthropod assemblage. Ecology, 81, 1120-1132.

Hanski, I. \& Koskela, H. (1977). Niche relations among dung-inhabiliting beetles. Oecología, 28, 203-231.

Humphrey, J. W., Hawes, C., Pearce, A. J., Ferris-Khan, R. \& Jukes, M. R. (1999). Relationships between insect diversity and habitat complexity in plantation forests. Forest Ecology and Management, 113, 11-21.

IUCN. (1994). Commision on National Parks and Protected Areas with the assistance of the World Conservation Monitoring Centre.

Jost, L. (2006). Entropy and diversity. Oikos, 113, 363-375.

Jost, L. (2007). Partitioning diversity into independent alpha and beta components. Ecology, 88, 2427-2439.

Kattan, G., Franco, P., Saavedra, C. A., Valderrama, C., Rojas, V., Osorio, D. \& Martínez, J. (2006). Spatial components of bird diversity in the Andes of Colombia: implications for designing a regional reserve system. Conservation Biology, 20, 1203-1211.

Lassau, S. A., Hochuli, D. F., Cassis, G. \& Reid, C. A. M. (2005). Effects of habitat complexity on forest beetle diversity: do functional groups respond consistently? Diversity and Distributions, 11, 73-82.

Legendre, P., Borcard, D. \& Peres-Neto, P. R. (2005). Analyzing beta diversity: partitioning the spatial 
variation of community composition data. Ecological Monographs, 75, 435-450.

Loreau, M., Naeem, S., Inchausti, P., Bengtsson, J., Grime, J. P. \& Hector, A. (2001). Biodiversity and ecosystem functioning: current knowledge and future challenges. Science, 294, 804-808.

Magurran, A. E. (2004). Measuring Biological Diversity. Oxford: Blackwell Publishing.

Mc.Cune, B. \& Mefford, M. J. (2002). Analysis of Ecological Communities. Gleneden Beach, Oregon, U.S.A.: MjM Software Design.

Mc.Cune, B. \& Mefford, M. J. (2011). PC-ORD, Multivariate Analysis of Ecological Data, Version 6. Gleneden Beach, Oregon, U.S.A.: MjM Software Design.

Meyke, E. (1999-2004). TAXIS 3.5-Taxonomical Information System. (Recuperado de www.bio-tools-net).

Moreno, C. E., Barragán, F., Pineda, E. \& Pavón, N. P. (2011). Reanálisis de la diversidad alfa: alternativas para interpretar y comparar información sobre comunidades ecológicas. Revista Mexicana de Biodiversidad, 82, 1249-1261.

Moschione, F. N. (2009). Diversidad y Conservación de Lepidópteros del Parque Nacional Los Cardones. APN-DRNO.59:09.

Orians, G. H. (1993). Endangered at what level? Ecological Applications, 3, 206-208.

Paknia, O. \& Pfeiffer, M. (2011). Hierarchical partitioning of ant diversity: implications for conservation of biogeographical diversity in arid and semi-arid areas. Diversity and Distributions, 17, 122-131.

Pearce, J. L., Venier, L. A., Eccles, G., Pedlar, J. \& McKenney, D. (2003). Influence of habitat and microhabitat on epigeal spider (Araneae) assemblages in four stand types. Biodiversity Conservation, 13, 1305-1334.

Peck, J. E. (2010). Multivariate Analysis for Community Ecologists: Step-by-Step using PC-ORD. Gleneden Beach, Oregon. USA: MjM Software Design.

Pinkus-Rendón, M. A., León-Cortés, J. L. \& Ibarra-Nuñez, G. (2006). Spiders diversity in a tropical habitat gradient in Chiapas, Mexico. Diversity and Distributions, 12, 61-69.

Purvis, A. \& Hector, A. (2000). Getting the measure of biodiversity. Nature, 405, 212-219.

Ramírez, M. J. (1999). Orden Araneae (clave para familias). (Recuperado de http://aracanologia.macn.gov. ar/biblio/Ramirez\%201999\%20clave.pdf.)

Rivera-García, E. \& Viggers-Carrasco, G. (1991). Estructura trófica de una comunidad de artrópodos epigeos, en un magueyal del bolsón de Mapimi, Dgo., México (Desierto Chihuahuense). Acta Zoológica Mexicana Nueva Serie, 48, 1-29.

Roig-Juñent, S., Flores, G., Claver, S., Debandi, G. \& Marvaldi, A. (2001). Monte Desert (Argentina): insect biodiversity and natural areas. Journal of Arid Environments, 47, 77-94.

Rojas, P. \& Fragoso, C. (2000). Composition, diversity, and distribution of a Chihuahuan Desert ant community (Mapimí, México). Journal of Arid Environments, 44, 213-227.

Roux, D. J, Nel, J. L., Ashton, P. J, Deacon, A. R., Moor, F. C. de, Hardwick, D., Hill, L., Kleynhans, C. J., Maree, G. A., Moolman, J. \& Scholes, R. J. (2008). Designing protected areas to conserve riverine biodiversity: Lessons from a hypothetical redesign of the Kruger National Park. Biological Conservation, 141, 100-117.

Sánchez, D. \& G. Amat-García. (2005). Diversidad de la fauna de artrópodos terrestres en el Humedal Jaboque, Bogotá-Colombia. Caldasia, 27, 311-329.

Sánchez, M. E. (2009). Línea de base de vegetación del Parque Nacional Los Cardones, Salta-Argentina. (Tesis Profesional IRMyMA). Universidad Nacional de Salta.

Stange, L. A., Terán, A. L. \& Willink, A. (1976). Entomofauna de la provincia biogeográfica del Monte. Acta Zoológica Lilloana, 32, 73-120.

SIB-APN. (2012). Sistema de Información sobre Biodiversidad-Administración de Parque Nacionales. Argentina. (Recuperado de http://www.sib.gov.ar/ area/APN*CD*LOS\%20CARDONES).

Tilman, D. (1999). The ecological consequences of changes in biodiversity: a search for general principles. Ecology, 80, 1455-1474.

Tóthmérész, B. (1998). On the characterization of scaledependent diversity. Abstracta Botanica, 22, 149-156.

Vane-Wright, R. I., Humphries, C. J. \& Williams, P. H. (1991). What to Protect-Systematics and the Agony of Choice. Biological Conservation, 55, 235-254.

Walther, B. A. \& Moore, J. L. (2005).Comparative performance of species richness estimation method. Parasitology, 116, 395-405.

Weeks, R. D. Jr. \& Holtzer, T. O. (2000). Habitat and Season in Structuring Ground-Dwelling Spider (Araneae) Communities in a Shortgrass Steppe Ecosystem. Environmental Entomology, 29, 1164-1172.

Whittaker, R. H. (1972). Evolution and measurement of species diversity. Taxon, 21, 213-251.

Whittaker, R. J., Willis, K. J. \& Field, R. (2001). Scale and species richness: towards a general, hierarchical theory of species diversity. Journal of Biogeography, 28, 453-470.

Woodcock, B. A., Watt, A. D. \& Leather, S. R. 2003. Influence of management type on Diptera communities of coniferous plantations and deciduous woodlands. Agriculture, Ecosystems \& Environment, 95, 443-452. 